for assessment. 656 contacts were vaccinated, 113 had immunity and 62 found to have active infection.

Discussion/Conclusion This audit clearly demonstrates the value and importance of partnership working to achieve successful public health outcomes well above targets set by national standards.

\section{NH4 SERVICE DEVELOPMENT FOR PEOPLE WITH HIV WHO HAVE COMORBID CONDITIONS USING EXPERIENCE- BASED CO-DESIGN METHODOLOGY}

${ }^{1}$ Eileen Nixon*, ${ }^{1,2}$ Vanessa Cooper, ${ }^{1,2}$ Elaney Youssef, ${ }^{2}$ Helen Smith, ${ }^{3}$ Glenn Robert, ${ }^{1,2}$ Martin Fisher. 'Brighton and Sussex University Hospitals, Brighton, UK; ${ }^{2}$ Brighton and Sussex Medical School, Brighton, UK; ${ }^{3}$ Kings College London, London, UK

\subsection{6/sextrans-2016-052718.49}

Background/introduction There is a need for evidence - based models of care to effectively manage the increasing numbers of the people with HIV (PWH) who have comorbid conditions. This study was part of an NIHR Programme Development Grant to inform the development of HIV services to meet the needs of an ageing population.

Aim(s)/objectives To explore the healthcare experiences of PWH who have comorbid conditions and the staff involved in their care to identify priorities for service improvement.

Methods Experience-based co-design methodology was used to understand the experiences of PWH accessing General Practice, HIV, Cardiology, Liver, Renal and Rheumatology services. Patients were recruited from the HIV clinic and staff purposively sampled from the service areas. Experiences were gathered through observation, diaries, audio and filmed interviews. Thematic analysis was undertaken and filmed patient interviews analysed for emotional touchpoints. Staff and patient feedback events were utilised to validate data and identify areas for service development. A joint staff and patient co-design event was held to agree shared priorities for future services.

Results 22 patients (with 110 comorbidities) and 18 staff were recruited. A composite film was produced from the patient interviews. Examples of touchpoints were communication, burden of appointments and repetition across services. Patients identified 6 areas for service improvement and staff identified 3. The agreed priorities for future service development were care co-ordination, shared medical records/results and systems to manage multiple appointments.

Discussion/conclusion Experience-based co-design methodology was effective in identifying future service models for PWH who have comorbid conditions.

\section{Section 4 Undergraduate oral Presentations}

\section{UG1 DIGITAL ANO-RECTAL EXAMINATION (DARE) AS ANAL CANCER SCREENING IN HIV POSITIVE MEN WHO HAVE SEX WITH MEN (HMSM) - IS IT ACCEPTABLE TO PATIENTS?}

${ }^{1}$ Tamara Lewis* ${ }^{2}$ Selvavelu Samraj, ${ }^{1,2}$ Raj Patel, ${ }^{2}$ Sangeetha Sundaram, ${ }^{1}$ School of Medicine, University of Southampton, Southampton, UK; ${ }^{2}$ Dept. of Sexual Health, Solent NHS Trust, Royal South Hants Hospital, Southampton, UK

10.1136/sextrans-2016-052718.50
Background/introduction Anal cancer is more common in HMSM than in HIV negative MSM or the general population. Tumours in HMSM tend to be larger and more advanced at diagnosis resulting in poorer prognosis. The European AIDS Clinical Society Guidelines recommend DARE with a screening interval of 1-3 years. However, this is only based on expert opinion. The benefit of such a strategy in a UK GUM managed HIV cohort is still unknown.

Aim(s)/objectives To assess acceptability of annual DARE to HMSM and establish patient experience of having DARE.

Methods From pre-published clinic lists covering the 8-week recruitment period, patients fitting the inclusion criteria (HMSM aged $\geq 35$ ) were invited to participate in the study when they attended clinic. Patients were asked to complete a questionnaire and invited to have DARE as part of their consultation.

Results Of the 43 patients invited into the study, 29 [67\%; 95\% confidence interval (CI) 53-81] proceeded to DARE. Principal reason for refusal of DARE was 'lack of time' and 'not feeling clean'. Of the 29 having DARE, 12 [41\%; 95\% CI 23-59] were found to have a previously unrecorded clinical abnormality. 5 [17\%; 95\% CI 3-31] required colorectal referral - 3 [10\%] for lesions suspicious of anal intraepithelial neoplasia. Outcomes of colorectal consultation are awaited. 100\% of respondents said they would have DARE again.

Discussion/conclusion Annual DARE is an acceptable addition to the routine care of HMSM. Pre-warning patients to expect DARE at a routine visit when it is due may further improve acceptability.

\section{UG2 GRINDR@ USE BY MEN WHO HAVE SEX WITH MEN (MSM) IS ASSOCIATED WITH HIGH RATES OF BACTERIAL SEXUALLY TRANSMITTED INFECTIONS}

${ }^{1}$ Samuel Owen*, 1,2Thomas Kurka, 1,2Daniel Richardson. 'Brighton \& Sussex Medical School, Brighton, UK; ${ }^{2}$ Brighton \& Sussex University Hospitals NHS Trust, Brighton, UK

\subsection{6/sextrans-2016-052718.51}

Background/introduction Mobile Phone 'apps' such as grindr (C) are becoming a more frequent and convenient way to meet sexual partners and may be a reason why sexually transmitted infections(STI) are increasing in MSM.

Methods From November 2015 to February 2016, a paper survey was distributed to MSM attending local sexual health services on acceptability of local service, including use of mobile phone applications to meet sexual partners. National Student Pride also used an online version of the survey.

Results 1186 MSM were included in the analysis of this survey. The median age was 26.8 years (18-89). 1026/1186 (86.5\%) self-identified as gay, $108 / 1186(9.1 \%)$ bisexual and $34 / 1186$ (2.9\%) straight. 918/1186 (77.4\%) were HIV-negative, 42/1186 (3.5\%) HIV-positive, 188/1186 (15.9\%) never tested, and 38/ 1186 (3.2\%) unknown status. 200/1186 (16.9\%) of respondents reported a bacterial STI within the past 12 months: 116/1186 (9.8\%) had gonorrhoea, 96/1186 (8.1\%) chlamydia and 26/1186 $(2.2 \%)$ syphilis. Reported use of grindr $@$ was: $372 / 1186$ $(31.4 \%)$ more than once/day, $168 / 1186(14.2 \%)$ more than once/week 124/1186 (10.5\%) more than once/month. Those who used grindr $\odot$ more than once per day reported having had gonorrhoea (62/372:16.7\%), chlamydia (50/372:13.4\%) and syphilis (16/372:4.3\%) in the past 12 months. 80/116 (70.0\%), $64 / 96(67 \%)$ and 16/26 (62\%) MSM who reported having gonorrhoea, chlamydia and syphilis in the past 12 months reported 
using grindr@ at least once a month. MSM were significantly more likely to report having gonorrhoea and chlamydia (but not syphilis) in the past 12 months using grindr $($ at least once per month compared to never using grindr(t-test $=2.79$; $\mathrm{p}=0.003), \quad(\mathrm{t}$-test $=2.20 ; \mathrm{p}=0.028), \quad(\mathrm{t}$-tst $=0.58$; $\mathrm{p}=0.565)$.

Discussion/conclusion Use of the mobile phone application grindr $($ ) is associated with acquisition of bacterial STIs. Public health interventions to reduce STI rates in MSM should include using appropriate social media.

\section{UG3 ARE GEOSOCIAL NETWORKING (GSN) APPS ASSOCIATED WITH INCREASED RISK OF STIS \& HIV: A SYSTEMATIC REVIEW}

${ }^{1}$ Matheus Almeida*, 1,2Jo Gibbs, 'Claudia Estcourt. 'Queen Mary University of London, London, UK; 'niversity College London, London, UK

\subsection{6/sextrans-2016-052718.52}

Background/introduction Geosocial networking (GSN) apps such as Tinder and Grindr provide new ways of finding sex partners. It is suggested that usage could be responsible for increased STI \& HIV transmission.

Aim(s)/objectives To systematically review published literature to determine whether geosocial app use is associated with increased sexual risk behaviours, current and/or previous STIs \& HIV.

Methods Search of PubMed, EMBASE and Google Scholar for studies involving women, men, men who have sex with men (MSM) and use of GSN apps for sex-seeking which reported risk factors for STIs \& HIV transmission, published from 2009 to March 2016, in English. Search terms were associated using at least one regarding GSN apps and a second regarding STIs or sexual risk behaviours. Quality was assessed using Critical Appraisal Skills Programme criteria.

Results 13 studies met inclusion criteria: 12 cross-sectional studies, 1 review. All were in MSM from urban USA, China, Taiwan, UK and Ireland. In total there were 11924 subjects (range 927184). 7 studies reported app use to be associated with increased unprotected anal intercourse (UAI); 2 studies showed no association. 3 studies showed association with previous STI diagnoses, although association with HIV diagnoses had mixed results. 4 studies reported high response rate for app-based recruitment.

Discussion/conclusion Use of GSN apps is associated with factors known to facilitate STI \& HIV transmission in MSM. Studies in heterosexuals are much needed. High uptake of some apprecruited studies suggests GSN apps could be useful platforms for sexual health promotion and targeted risk reduction strategies.

\section{UG4 NON-SPECIFIC URETHRITIS: CAN WE BE A LITTLE MORE SPECIFIC?}

${ }^{1}$ Genevieve Hirst*, ${ }^{2}$ Daniel Richardson, ${ }^{2}$ Suneeta Soni. ${ }^{1}$ Peninsula Medical School, Truro, UK; ${ }^{2}$ Brighton and Sussex University Hospitals NHS Trust, Brighton, UK

\subsection{6/sextrans-2016-052718.53}

Background/introduction The causes of non-specific urethritis (NSU) in men are many and in GUM clinics, evidence for Chlamydia trachomatis (CT) and Neisseria gonorrhoeae (GC) is routinely sought. Mycoplasma genitalium (MG) accounts for 5-33\% of urethritis but is not routinely tested for in the UK. There is growing concern that widespread use of $1 \mathrm{~g}$ Azithromycin is leading to macrolide resistance in many organisms including MG.

Aim(s)/objectives To describe the current management of men with confirmed urethritis and their outcomes.

Methods Men with diagnoses of NSU from January to July 2015 were identified. Data were collected from electronic patient records. $\mathrm{p}$ values were obtained using chi-square test.

Results 254 cases of NSU were identified, median age 30 (range 16-69 years). 181/254 (71\%) heterosexual, 73/254 (29\%) MSM, 21/254 (8\%) HIV-positive. Rates of urethral CT and GC were $15 \%(n=40)$ and $1 \%(n=2)$ respectively. $21 / 254(8 \%)$ had persistent dysuria or discharge; $15 / 21$ of those were tested for MG; MG detected in 5/15 (33\%). Pathogens were identified in $17 \%$ of cases and heterosexual men were more likely to have pathogen-positive urethritis than MSM $(p=0.02)$. First line treatment: 93\% $1 \mathrm{~g}$ Azithromycin, 2.8\% doxycycline $100 \mathrm{mg}$ bd $7 / 7$.

Discussion/conclusion For the majority of NSU cases, no bacterial cause was identified yet these men were all prescribed antibiotics. MG was detected in a third of persistent NSU cases but may account for more as $1 \mathrm{~g}$ Azithromycin is enough to partially resolve symptoms but likely cause antimicrobial resistance. More effort should be made to determine the cause of urethritis in men so that appropriate antibiotics can be given where necessary.

\section{UG5 SH:24 - USER PERSPECTIVES ON AN ONLINE SEXUAL HEALTH SERVICE}

${ }^{1}$ Harriet Pittaway*, 'Sharmani Barnard, ${ }^{2}$ Emma Wilson, ${ }^{1}$ Paula Baraitser. 'King's Centre for Global Health, King's College London, London, UK; ${ }^{2}$ Department of Population Health, Faculty of Epidemiology and Population Health, London School of Hygiene and Tropical Medicine, London, UK

\subsection{6/sextrans-2016-052718.54}

Background/introduction The London Boroughs of Lambeth and Southwark have high levels of sexual health need and services are overstretched. SH:24 offers online testing for chlamydia, gonorrhoea, HIV and syphillis in Lambeth and Southwark, and the 'GetTested' randomised controlled trial evaluates its effectiveness.

Aim(s)/objectives This study aimed to document user views on clinic-based and online services.

Methods We analysed qualitative data from a follow-up questionnaire of the GetTested trial of 1337 participants, which included the following question: 'In your opinion, how could we improve the experience of getting a test from a sexual health service?' This data was quantitatively analysed against baseline characteristics to generate descriptive statistics. A thematic analysis of the free text responses was performed.

Results Three key themes were identified: interaction with services; ease of use and experienced stigma. A subjective variable was developed to describe whether users needs were met. More participants reported the online service as meeting their needs than the clinic service. Areas needing improvement identified within the clinic arm were: Information prior to service use, Improved confidentiality \& Waiting times. Areas needing improvement identified within the online arm were: Lack of personal contact, Difficulty with the self-sampling process, Confidence in ability to self-test.

Discussion/conclusion The problems identified with face-to-face services are overcome by online services and vice versa. In order 